\title{
Cloning and Expression of cDNAs for Biosynthesis of Very-long-chain Fatty Acids, the Precursors for Cuticular Wax Formation, in Carnation (Dianthus caryophyllus L.) Petals
}

\author{
Masaya Kawarada ${ }^{1}$, Yoshihiro Nomura ${ }^{1}$, Taro Harada ${ }^{1 * *}$, Shigeto Morita ${ }^{1,2}$, \\ Takehiro Masumura ${ }^{1,2}$, Hiroyasu Yamaguchi ${ }^{3}$, Koji Tanase $^{3}$, \\ Masafumi Yagi ${ }^{3}$, Takashi Onozaki ${ }^{3}$ and Shigeru Satoh ${ }^{1,2 *}$ \\ ${ }^{1}$ Graduate School of Life and Environmental Sciences, Kyoto Prefectural University, Kyoto 606-8522, Japan \\ ${ }^{2}$ Kyoto Prefectural Institute of Agricultural Biotechnology, Seika-cho, Kyoto 619-0244, Japan \\ ${ }^{3}$ Ornamental Plants Research Division, NARO Institute of Floricultural Science, Tsukuba 305-8519, Japan
}

\begin{abstract}
The cuticle, composed of cutin and associated waxes, probably acts as a barrier against water evaporation from the epidermal surface of flower petals. Cuticle formation begins with the biosynthesis of very-long-chain fatty acids (VLCFAs), catalyzed by a fatty acid elongase complex in epidermal cells. In the present study, cDNAs were cloned and analyzed for three enzymes (DcKCR1, DcHCD1, and DcECR1). Combined with the previously obtained cDNA for DcKCS1, the present study completes the identification of cDNAs for the fatty acid elongase complex in 'Light Pink Barbara' carnation for the first time. DcKCS1 transcripts were accumulated at flower opening stage (Os) 2 through Os 6 (full opening stage) with slight changes, but decreased markedly at senescence stage (Ss) 2 and Ss 4. Also, transcripts for DcKCR1, DcHCD1, and DcECR1 were present in considerable amounts during flower opening stages from $\mathrm{Os} 2$ to $O s$. These findings suggested that the expressions of four genes are active during flower opening stage, which is concomitant with the expansion growth in petals requiring rapid formation of a waxy cuticle. Cut flowers of 'Miracle Rouge' carnation have an extremely long vase-life of about three weeks. The cuticle layer on the epidermal cells of 'Miracle Rouge' petals was thinner than that of 'Light Pink Barbara' petals, and 'Miracle Rouge' flowers had a depressed expression of DcKCS1, DcKCR1, and DcHCD1 in petals. These findings suggested that the prolonged vase-life of 'Miracle Rouge' flowers is not related to cuticle formation.
\end{abstract}

Key Words: carnation, cuticular wax, fatty acid elongase, flower opening, petal, very-long-chain fatty acids.

\section{Introduction}

The plant cuticle is an extracellular lipid structure deposited over the surface of leaves, stems, and petals, etc. of land plants, and prevents water loss, pathogen invasion, and herbivorous insect attack (Riederer and Schreiber, 2001). It is composed of cutin polymer matrix and waxes, produced and secreted by epidermal cells.

Received; December 1, 2012. Accepted; January 22, 2013.

This study was supported financially by a Grant-in-Aid (24580050 to S.S.) for Scientific Research from the Japan Society for the Promotion of Science.

* Corresponding author (E-mail: ssatoh@kpu.ac.jp).

** Present address: Horticultural Institute, Ibaraki Agricultural Center, Kasama 319-0292, Japan.
Cutin forms extensive polymeric structures, composed mainly of $\omega$ - and mid-chain mono- and dihydroxy and epoxy $\mathrm{C}_{16}$ and $\mathrm{C}_{18}$ fatty acids. The hydroxyl groups of the fatty acids allow ester links to be formed and so build up a polyester structure (Harwood, 1997). Cuticular wax comprises a looser mixture of aliphatic compounds, mostly very-long-chain fatty acid (VLCFA) derivatives. The actual components are hydrocarbons (alkanes) in a high proportion among total components, followed by monoesters of long-chain alcohols and fatty acids, and very-long-chain alcohols (mainly $\mathrm{C}_{22}-\mathrm{C}_{34}$ ) and verylong-chain saturated fatty acids (Harwood, 1997). For instance, in snapdragon, the major components of petal wax are n-alkanes $(29.0-34.3 \%)$, followed by methylbranched alkanes (23.6-27.8\%) and hydroxyl esters (12.0-14.0\%) (Goodwin et al., 2003). 
VLCFA precursors of wax production are synthesized in epidermal cells by the multienzyme fatty acid elongase (FAE) complex on the endoplasmic reticulum (Kunst and Samuels, 2009). Using $C_{16}$ and $C_{18}$ fatty acids and malonyl-coenzyme $\mathrm{A}(\mathrm{CoA})$ as substrates, FAE mediates successive reactions catalyzed by a $\beta$-ketoacyl-CoA synthase (KCS), a $\beta$-ketoacyl-CoA reductase (KCR), a $\beta$-hydroxyacyl-CoA dehydratase (HCD), and an enoylCoA reductase (ECR) (Fig. 1). These four reactions perform a reiterative cycle to produce saturate VLCFAs with $24-36$ carbon atoms. The KCS is the rate-limiting enzyme of fatty acid elongation and determines the length of the final acyl-CoA product (Denic and Weissman, 2007; Millar and Kunst, 1997). Recent studies in Arabidopsis thaliana have identified a set of genes (cDNAs) for the four enzymes involved in FAE; CER6, a FAE1-type KCS, for KCS (Millar et al., 1999), KCRl for KCR (Beaudoin et al., 2009), ASTICCINO2 (PAS2) for $H C D$ (Bach et al., 2008), and CER10 for ECR (Zheng et al., 2005). In addition to PAS2, HCD1 has been suggested to act in reduction in Arabidopsis (Raffaele et al., 2008).

Flower opening results from the expansion growth of petal cells, which in turn is forced by increased turgor of the cells. If turgor is insufficient during flower opening, the petal cells will not expand properly, resulting in a failure of flower opening. Also, it will cause petal wilting and flower deterioration in cut fullyopened flowers. Water loss from petals is determined by non-stomatal transpiration through the cuticle on the epidermal cell surface. As described above, cuticle wax acts as the major lipid barrier against water loss from petals. Without an intact wax layer, flower petals are vulnerable to water loss, resulting in a failure of flower opening or the onset of wilting in fully-opened flowers; therefore, cuticle wax formation is important to support normal flower opening and to keep flowers open.

Ethylene plays a primary role in the senescence of cut carnation flowers (Abeles et al., 1992; Borochov and

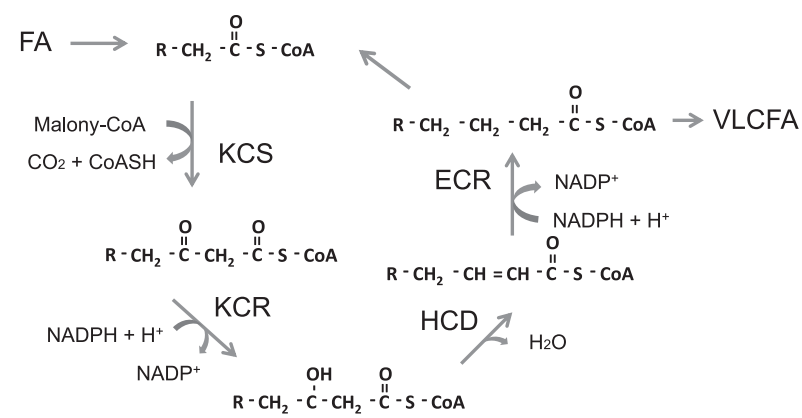

Fig. 1. Cyclic reaction for VLCFA synthesis by a fatty acid elongase complex. cDNAs for KCR, HCD and ECR were obtained as $D c K C R 1, D c H C D 1$, and DcECR 1 from carnation petals in the present study, but $D c K C S 1$ cDNA was found previously by Harada et al. (2010). FA, fatty acid; KCS, $\beta$-ketoacyl-CoA synthase; KCR, $\beta$-ketoacyl-CoA reductase; HCD, $\beta$-hydroxyacyl-CoA dehydratase; ECR, enoyl-CoA reductase; VLCFA, very-long-chain fatty acid.
Woodson, 1989; Reid and $\mathrm{Wu}, 1992)$. In flowers undergoing natural senescence, ethylene is first produced from the gynoecium and the evolved ethylene induces autocatalytic ethylene production in petals, resulting in their wilting (Shibuya et al., 2000; ten Have and Woltering, 1997). Petal wilting is caused by desiccation of petal tissues, which in turn is caused by transpiration through cuticle layers on the surface of the petals. Cuticle thickness probably varies among carnation cultivars; therefore, we could anticipate that cuticle formation, especially wax formation, eventually affects the senescence of cut carnation flowers through the regulation of water loss by cuticle transpiration.

Recently, Harada et al. (2010) identified a cDNA (DcKCS1, DDBJ acc. no. AB517655) for KCS in carnation, and showed accumulation of its transcript in the petals of carnation flowers undergoing flower opening. In the present study, to gain more insight regarding the relationship, if any, between petal wilting during flower senescence and cuticle characteristics in carnation flowers, we aimed to clone cDNAs for other enzymes of FAE involved in the biosynthesis of VLCFAs and analyze the expression of their genes in flower tissues, especially petals, of opening carnation flowers. In addition, we compared the expression of these genes in petals among three cultivars, 'Light Pink Barbara' and 'Excerea' with ordinary ethylene production and senescence characteristics, and 'Miracle Rouge' with undetectable ethylene production and long vase-life of flowers. The 'Miracle Rouge' cultivar was recently generated by cross-breeding at NARO Institute of Floriculture Science (Onozaki et al., 2006; Tanase et al., 2008).

\section{Materials and Methods}

\section{Plant materials and sample preparation}

Cut flowers of the spray carnation (Dianthus caryophyllus L. 'Light Pink Barbara') were harvested when the first flower out of five to six flower buds on a stem was nearly open at the nursery of a commercial grower in Miyagi prefecture. The flowers were transported dry to the laboratory at Kyoto Prefectural Institute of Agricultural Biotechnology in Kyoto prefecture the day after harvest. Depending on the season when experiments were performed, flowers of the same cultivar were obtained similarly from commercial nurseries in Hyogo prefecture and Nagano prefecture. They were placed in plastic containers with their cut end in water under continuous light from white fluorescent lamps $\left(14 \mu \mathrm{mol} \cdot \mathrm{m}^{-2} \cdot \mathrm{s}^{-1} \mathrm{PPFD}\right)$ at $23^{\circ} \mathrm{C}$.

The flowers of the standard carnation cultivars, 'Excerea' and 'Miracle Rouge', which have only one flower on a stem, were harvested at the nursery of NARO Institute of Floricultural Science, Tsukuba, Japan. Immediately after harvesting the flowers, they were separated into flower parts and stored at $-80^{\circ} \mathrm{C}$. The frozen floral tissues were then transported to Kyoto 
Prefectural Institute of Agricultural Biotechnology in Kyoto prefecture and used for RNA extraction.

The flower-opening process was separated into 6 stages (Os 1 to Os 6), as described in Harada et al. (2010). The flower senescence process was separated into 4 stages (Ss 1 to $\mathrm{Ss} 4$ ), as described previously (Morita et al., 2011). The profiles of flower opening and senescence were shown previously (Fig. 2 in Morita et al., 2011).

For cloning the respective cDNAs, ten petals detached from flowers at Os 5 were sampled from 'Light Pink Barbra' flowers. For gene expression analysis, ten outermost petals were detached from 5 flowers at the respective stages, combined and mixed to make one sample (making a sample of 50 petals), and stored at $-80^{\circ} \mathrm{C}$ for extraction of RNA. A series of samples collected at each stage during flower opening and senescence was prepared and used for RT-PCR and realtime RT-PCR analyses. Other tissues, leaf, stem, calyx, receptacle, ovary, and style, were detached from plants with fully opened 'Light Pink Barbara' flowers and stored as above. In carnation flowers, the stigma is along the surface of the distal half of the style (rather than a distinct structure as in other flowers); therefore, the term 'style' was used to describe the style plus stigma in this study.

\section{RNA extraction}

Total RNA was isolated by the phenol-chloroform method from carnation tissues, as described in Harada et al. (2005). Briefly, a sample of about $1 \mathrm{~g}$ frozen petals, or other tissues, was pulverized in liquid $\mathrm{N}_{2}$ with a mortar and pestle, and extracted with $5 \mathrm{~mL}$ extraction buffer [1\% SDS, $50 \mathrm{mM}$ Tris- $\mathrm{HCl}$ ( $\mathrm{pH} 7.5$ ), $50 \mathrm{mM}$ EDTA] plus an equal volume of water-saturated phenol. Total RNA was recovered from the supernatant after centrifugation, purified by treatment with phenol-chloroform solution $(1: 1 \mathrm{v} / \mathrm{v})$ followed by chloroform, and finally precipitated with isopropanol at $-20^{\circ} \mathrm{C}$.

PCR cloning of cDNA of $\beta$-ketoacyl-CoA reductase (KCR), $\beta$-hydroxyacyl-CoA dehydratase (HCD), and enoyl-CoA reductase (ECR)

To obtain full-length composite cDNAs for carnation $K C R, H C D$, and $E C R$, we used a nearly identical strategy in which three partial-length cDNAs were obtained by ordinary RT-PCR, 3'-RACE (rapid amplification of cDNA end, Frohman et al., 1988) and 5'-RACE techniques, and then the cDNAs were combined to make a composite cDNA. Then, to confirm complete nucleotide sequences, full-length cDNAs were amplified with primers derived from both ends of the composite cDNAs and total RNAs obtained from carnation petals. The forward and reverse primer pairs for PCR to obtain partial-length cDNAs and full-length cDNAs and sizes (in base pairs) of amplificates and composite cDNAs are summarized in Table 1.

For cloning carnation $K C R$ cDNA, we utilized the partial nucleotide sequence (acc. no. DK999722, 275 bp), which was previously reported by Harada et al. (2010), as an initial cDNA fragment. For cloning carnation $H C D$ and $E C R$ cDNAs, we cloned one partial-length cDNA each for the respective genes, with total RNA obtained from opening carnation petals (Os 5) and the sets of degenerate forward and reverse primers (Table 1), which were derived from sequences of $H C D$ and $E C R$ cDNAs reported in public databases. RT-PCR was performed according to the standard procedure with necessary optimization. The PCR products were cloned into pGEM-T Easy Vector (Promega, Madison, WI, USA)

Table 1. Primers used for PCR cloning and RT-PCR and real-time PCR analyses.

\begin{tabular}{|c|c|c|c|c|}
\hline \multicolumn{2}{|c|}{ Genes, purpose and target } & Foward (primer name; sequence, $5^{\prime}$ to $3^{\prime}$ ) & Reverse (primer name; sequence, $5^{\prime}$ to $3^{\prime}$ ) & $\begin{array}{c}\text { Amplificate } \\
\text { (bp) }\end{array}$ \\
\hline \multicolumn{5}{|c|}{ PCR cloning } \\
\hline \multirow[t]{2}{*}{ DcKCRl } & 3'-RACE & DcKCR1-F1; GTGTGCCTGAGAGCATCATCG & 3'RACE primer; GAAGAATTCGCGGCCGCAGGAA & 329 \\
\hline & 5'-RACE & 5'RACE primer; CGACTGGAGCACGAGGACACTGA & DcKCR1-R1; GTCTGTTCAATCCGCTGGCG & 1072 \\
\hline \multicolumn{2}{|c|}{ DcHCDI First round PCR } & HCD-F; AARTTCTTCTCCAAYGGMTTYGAYCT & HCD-R; AGNCCRATRTCMACYTCRCTCATRTA & 257 \\
\hline & 3'-RACE & DcHCD1-F1; CTTCGCTGGGCCCAATCC & 3'RACE primer; GAAGAATTCGCGGCCGCAGGAA & 648 \\
\hline \multicolumn{2}{|c|}{ DcECRI First round PCR } & ECR-F; TAYTAYTGGTGYTTYCAYTAYTTYAA & ECR-R; AAYTAYACNACNGAGATYTAYCARTGG & 351 \\
\hline & 3'-RACE & DcECR1-F1; GGGTATCAGATTCCTAAGGGATTCTTG & 3'RACE primer; GAAGAATTCGCGGCCGCAGGAA & 371 \\
\hline & 5'-RACE & 5'RACE primer; CGACTGGAGCACGAGGACACTGA & DcECR1-R1; AATCCCTTAGGAATCTGATACCCG & 828 \\
\hline \multicolumn{5}{|c|}{ Full length cDNA amplification } \\
\hline \multicolumn{2}{|l|}{$D c K C R l$} & DcKCR1-F0; ATGGTGCATTGTTGTATAATAGAAAGGCT & DcKCR1-R0; GCACGACCTAAGTCGAGATAATATGG & 1201 \\
\hline \multicolumn{2}{|l|}{$\mathrm{DcHCDl}$} & DcHCD1-F0; CCAACTCACCCAAAAAAACACCACC & DcHCD1-R0; ACTAAAGCATTCATTCAACACCCGAC & 898 \\
\hline \multicolumn{5}{|c|}{ Real-time PCR analysis, RT-PCR amplification } \\
\hline \multicolumn{2}{|c|}{$\operatorname{DcKCSI}$} & DcKCS1-rF1; GCATCGATTTGGTAACACGTCTAGTAG & DcKCS1-rR1; GGATAGAGATCAATCTCTTCATTCCACG & 204 \\
\hline \multicolumn{2}{|l|}{$D c K C R 1$} & DcKCR1-rF1; GAGCTGTCAAAAAATGCTCGTCAATGTC & DcKCR1-rR1; GCACGACCTAAGTCGAGATAATATGG & 219 \\
\hline \multicolumn{2}{|l|}{$\mathrm{DcHCD} 1$} & DcHCD1-rF1; GGTGAGATTAGGAAGGGTTTGTACC & DcHCD1-rR1; GACTAAAGCATTCATTCAACACCCGAC & 198 \\
\hline \multicolumn{2}{|l|}{$D c E C R I$} & DcECR1-rF1; CGACGCCTGAAGAAGTTATTTGACG & DcECR1-rR1; CAGCAACTGAAACTTGATTCATTCTGTAGC & 203 \\
\hline \multicolumn{2}{|l|}{$D c U b q 3-7$} & Ubq3F; GTTGTTGGTTTCAGGGCTGGTTTG & Ubq3R; CTACGGTAATTGAGAATTCACACCGAAATG & 178 \\
\hline
\end{tabular}


for sequencing, and the nucleotide sequence was obtained. Using the nucleotide sequences, sequencespecific primers were designed. Then, for cloning $K C R$ cDNA, as a representative, a 3'-downstream cDNA of $329 \mathrm{bp}$ was obtained by $3^{\prime}$-RACE with the forward DcKCR1-F1 primer and the reverse 3'RACE primer. Finally, a 5'-upstream cDNA of 1072 bp was obtained by 5 '-RACE with the forward 5'RACE primer and the reverse DcKCR1-R1 primer. The three partial cDNAs were reconstituted to make the composite cDNA. Then, to confirm complete nucleotide sequences, full-length cDNA (1201 bp) was amplified from total RNA obtained from opening carnation petals as templates with primers derived from both ends of the composite cDNA (DcKCR1-F0 primer; DcKCR1-R0 primer). The nucleotide sequence of the cDNA was obtained, and the complete sequence was confirmed to be identical to the composite sequence. For cloning $H C D$ and ECD DNAs, similar procedures were employed with the primers described in Table 1. Nucleotide sequences were edited and analyzed by GENETYX-WIN and a homology search was performed using the BLASTX program available from the DDBJ website.

\section{Real-time RT-PCR}

Real-time RT-PCR for the quantification of transcripts for DcKCS1, DcKCR1, DcHCD1, and DcECR1 and DcUbq3-7 (Nomura et al., 2012) was conducted using the LightCycler FastStart DNA Master SYBR Green I Kit and the LightCycler Instrument (Roche Diagnostics K.K., Tokyo, Japan). cDNA was synthesized using ReverTra Ace (TOYOBO, Osaka, Japan) according to the manufacturer's instructions. The primer sets for realtime RT-PCR for respective genes are shown in Table 1. PCR conditions were initial heating for $10 \mathrm{~min}$ at $95^{\circ} \mathrm{C}$ followed by 40 cycles of $5 \mathrm{~s}$ at $95^{\circ} \mathrm{C}, 5 \mathrm{~s}$ at $53^{\circ} \mathrm{C}$, and $8 \mathrm{~s}$ at $72^{\circ} \mathrm{C}$, in which the extension time was adopted according to the length of amplificates ( $1 \mathrm{~s}$ per $25 \mathrm{mer}$ ). The absolute transcript level was calculated using a dilution series of a target sequence on LightCycler Software Ver. 3.5.3 (Roche Diagnostics). Three independent RNA preparations per stage were used for analyses, and data are shown as the mean $\pm \mathrm{SE}$ of the three samples for each stage.

\section{$R T-P C R$}

Total RNAs were isolated from petals at Os 6 of three carnation cultivars, as described above. Reverse transcription was performed with ReverTra Ace using an oligo dT primer. The cDNAs were amplified with Taq DNA polymerase under the conditions of $96^{\circ} \mathrm{C}$ for 3 min followed by 38 cycles of $30 \mathrm{~s}$ at $96^{\circ} \mathrm{C}, 30 \mathrm{~s}$ at $53^{\circ} \mathrm{C}$, and $1 \mathrm{~min}$ at $72^{\circ} \mathrm{C}$. DcUbq3-7 cDNAs (Nomura et al., 2012) were also amplified as standard under the conditions of $94^{\circ} \mathrm{C}$ for $3 \mathrm{~min}$ followed by 36 cycles of $30 \mathrm{~s}$ at $94^{\circ} \mathrm{C}, 30 \mathrm{~s}$ at $53^{\circ} \mathrm{C}$, and $1 \mathrm{~min}$ at $72^{\circ} \mathrm{C}$. The primer sets for amplification were the same as those used for real-time RT-PCR. The amplificates were separated by agarose gel electrophoresis, stained with ethidium bromide and quantified with KODAK 1D Image Analysis Software (Eastman Kodak, Rochester, NY, USA).

\section{Measurement of cuticle thickness}

For measurement of the thickness of cuticles, carnation petals at Os 6 were detached from outer whorls of fully opened flowers, and square segments $\left(5 \times 5 \mathrm{~mm}^{2}\right)$ were cut out from the central part of petal blades using a razor blade. The petal segments were embedded in 3\% $(\mathrm{w} / \mathrm{v})$ caboxymethylcellulose (CMC) gel and frozen in cold hexane $\left(-75^{\circ} \mathrm{C}\right)$. Cryostat sections of $10 \mu \mathrm{m}$ thickness were prepared using the Cryo-Film Transfer Kit (Fintec Co. Ltd., Tokyo, Japan) (Kawamoto, 2003). The film sections were put on slide glasses, and CMC in the section was removed by adding a water drop and then removing it. Then, the sections were stained with Sudan Black (saturated in $70 \%$ ethanol) for $30 \mathrm{~min}$ at room temperature. The excess staining solution was removed by adding a drop of $70 \%$ ethanol and then removing it. The sections were mounted with glycerin under a cover film. The sections were inspected under a microscope and photographed. Photographs were magnified on a computer display, and cuticle thickness was determined by a ruler for ten epidermal cells on the adaxial side.

\section{Statistical analysis}

Statistical analysis was carried out by Tukey's multiple range test using an on-line statistical analysis program MEPHAS (http://www.gen-info.osaka-u.ac.jp/testdocs/ tomocom/).

\section{Results and Discussion}

Cloning and characterization of cDNAs for components of fatty acid elongase, KCR, HCD, and ECR, in carnation flowers

One cDNA each of putative genes for three components of fatty acid elongase complex was isolated from total RNAs obtained from opening petals of 'Light Pink Barbara' carnation using a combination of RT-PCR, 5'-RACE and 3'-RACE techniques. We named the original genes for isolated cDNAs in carnation (Dianthus caryophyllus) as follows and deposited in DDBJ; DcKCRl (acc. no. AB750616), DcHCDl (acc. no. AB750617), and DcECRI (acc. no. AB750618).

$D c K C R 1$ cDNA was 1201 bp in length, having an entire open reading frame (ORF) of $966 \mathrm{bp}, 1$-bp long 5'-UTR and 234-bp long 3'-UTR. The putative DcKCR1 consisted of 321 amino acids corresponding to a molecular mass of $35.8 \mathrm{kDa}$. DcKCR1 protein had an amino-acid sequence corresponding to that of the NADB-Rossman superfamily at amino-acid numbers 54-229, and amino-acid residues for an active site and an $\mathrm{NAD}(\mathrm{P}) \mathrm{H}$ binding site (Fig. 2A). DcKCR1 had 

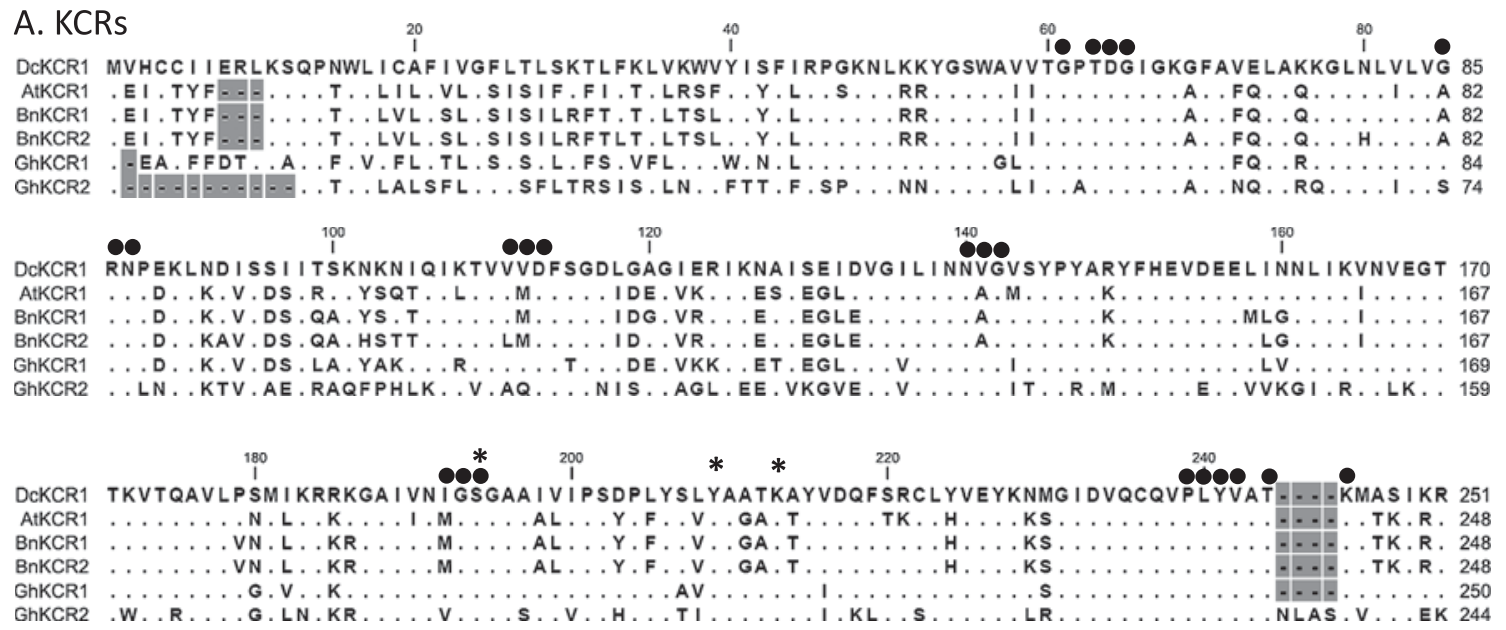

2800

DCKCR1 SSFFVPSTEGYAKAGIRHIGYEPRCTPYWPHSLIWG IASCVPESIIDAWRLRFCLAIRKRGOLKDSRE-KKD 321

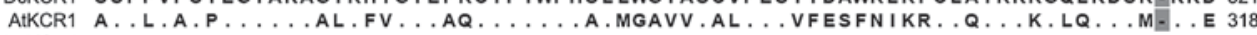

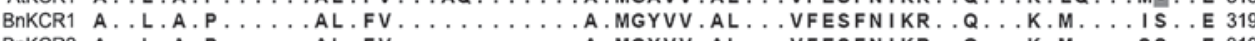

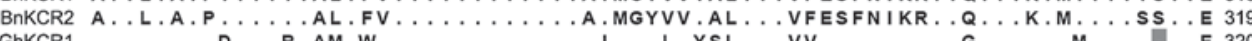

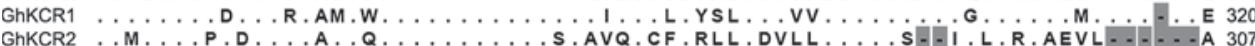

\section{B. HCDs}

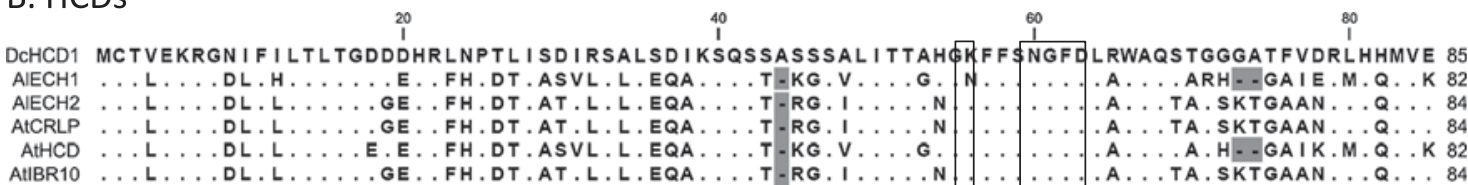

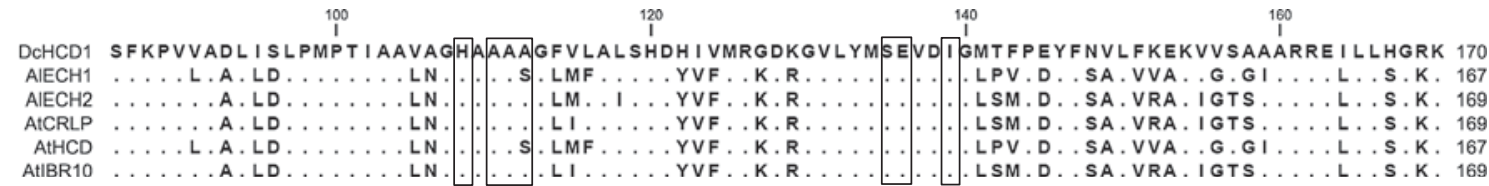

180

200

${ }_{120}^{220}$

${ }^{240}$

DCHCD1 LKAAEAKEFGVVDEVGKDGEDVVVAKAVGLATELVKRKWNGEVYGEIRKGLYPNLCLELGLSKRTVVVPRL 240

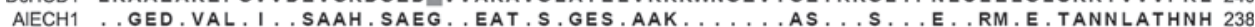

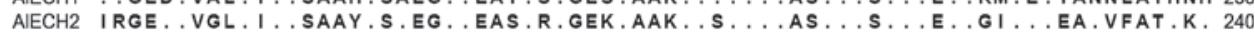

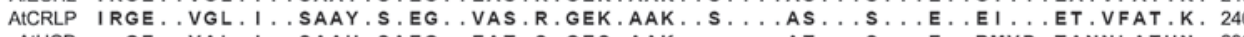

AtHCD . GE . VAL. I . SAAH.SAEG. . EAT.S.GES.AAK . . . . . A . . . . . E . RMVD.TANNLATHN. 238

AtIBR10 IRGE..VGL. I . SAAY.S.EG..VAS.R.GEK.AAK.. . . . . . . . . . E. . I . . ET.VFAT.K. 240

Fig. 2. Alignment of amino-acid sequences of DcKCR1 (A), DcHCD1 (B), and DcECR1 (C) from carnation and other plant sources. Common amino acids are shown by dots and gaps by hyphens on a grey background. A, closed circles at the top of alignments show amino-acid residues for the $\mathrm{NAD}(\mathrm{P}) \mathrm{H}$ binding site and stars show amino-acid residues for an active site. $\mathrm{B}$, amino-acid residues for substrate binding sites are enclosed by boxes. C, triangles show amino-acid residues for a charged pocket and amino-acid residues enclosed by boxes are those for a hydrophobic patch. KCRs: DcKCR1 (acc. no. AB750616, carnation, this study), AtKCR1 (acc. no. NP_001234455, Arabidopsis thaliana), BnKCR1 (acc. no. AAO43448.1, Brassica napus), BnKCR2 (acc. no. AAO43449.1, B. napus), GhKCR1 (acc. no. AAY23354.1, cotton), GhKCR2 (acc. no. AAY23355.1, cotton). HCDs: DcHCD1 (acc. no. AB750617, carnation, this study), AlECH1 (acc. no. XP_002868281.1, Arabidopsis lyrata subsp. lyrata), AlECH2 (acc. no. XP_002868281.2, A. lyrata subsp. lyrata), AtCRLP (acc. no. AAM63923, A.thaliana), AtHCD (acc. no. NP_193180.1, A.thaliana), AtIBR10 (acc. no. NP_193179.1, A. thaliana). ECRs: DcECR1 (acc. no. AB750618, carnation, this study), AlECR (acc. no. XP_002878031.1, A. lyrata subsp. lyrata), AtECR (acc. no. NP_191096.1, A. thaliana), BnECR (acc. no. ACR56738, B. napus), GhECR (acc. no. ABV60089, cotton), PaECR (acc. no. ADK74340, Phalaenopsis amabilis).

homologies of $64.1 \%$ to AtKCR1 (acc. no. NP 001234455 , Arabidopsis thaliana), $65.3 \%$ to BnK̄CR1 (acc. no. AAO43448.1, Brassica napus), $64.3 \%$ to BnKCR2 (acc. no. AAO43449.1, B. napus), 75.6.1\% to GhKCR1 (acc. no. AAY23354.1, cotton), and $56.5 \%$ to GhKCR2 (acc. no. AAY23355.1, cotton).

DcHCDl cDNA was 898 bp in length, with an ORF of $723 \mathrm{bp}, 61$-bp long 5'-UTR and 114-bp long 3'-UTR.
The putative DcHCD1 protein consisted of 240 amino acids corresponding to a molecular mass of $26.0 \mathrm{kDa}$. DcHCD1 had an amino-acid sequence corresponding to that of the crotonase/enoyl-coenzyme A ( $\mathrm{CoA})$ hydrolase superfamily at amino-acid numbers 2-203 and aminoacid residues for the substrate binding site (Fig. 2B). DcHCD1 had homologies of $57.2 \%$ to AlECH1 (enoylCoA hydratase/isomerase, acc. no. XP_002868281.1, 


\section{ECRs}
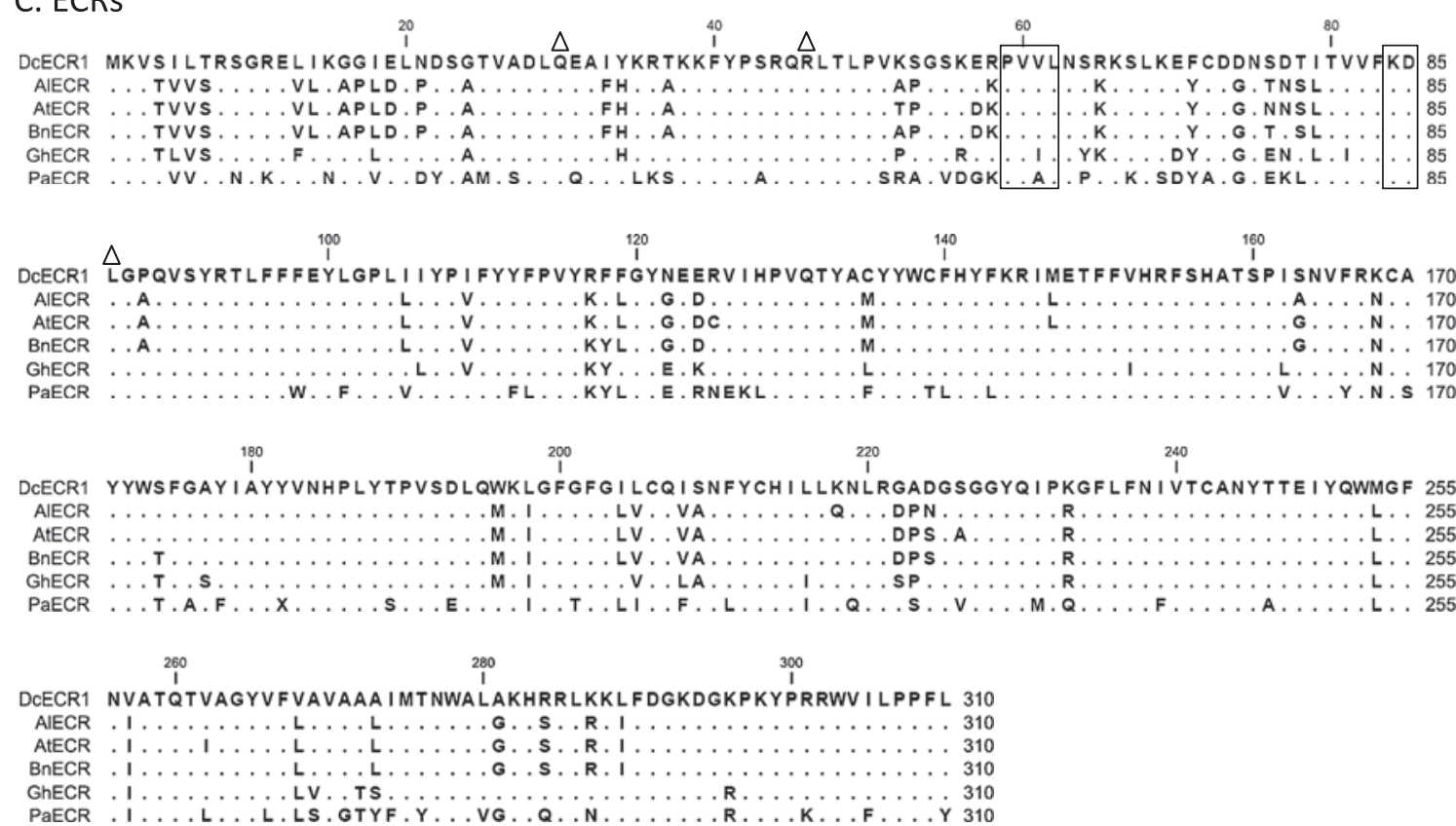

Fig. 2. Continued

Arabidopsis lyrata subsp. lyrata), $57.7 \%$ to AlECH2 (enoyl-CoA hydratase/isomerase, acc. no. XP 002868281.2, A. lyrata subsp. lyrata), 58.1\% to AtCRLP (carnitine racemase like protein, acc. no. AAM63923, A. thaliana), 56.9\% to AtHCD (acc. no. NP_193180.1, A. thaliana), and $58.1 \%$ to AtIBR10 (acc. no. NP 193179.1, A. thaliana).

DcECR 1 cDNA was 1175 bp in length, with an ORF of $933 \mathrm{bp}, 123$-bp long 5'-UTR and 119-bp long 3'-UTR. The putative DcECR1 consisted of 310 amino acids corresponding to a molecular mass of $36.1 \mathrm{kDa}$. DcECR1 had a UBQ-like domain of TSC13, an amino-acid sequence resembling that of 2 -oxo-5- $\alpha$-steroid 4dehydrogenase, amino-acid residues for making a charged pocket and those for a hydrophobic patch (Fig. 2C). DcECR1 had homologies of $82.3 \%$ to AlECR (acc. no. XP 002878031.1, A. lyrata subsp. lyrata), 81.3\% to AtECR (acc. no. NP 191096.1, A. thaliana), $82.3 \%$ to BnECR (acc. no. ACR56738, B. napus), $84.5 \%$ to GhECR (acc. no. ABV60089, cotton), $69.6 \%$ to PaECR (acc. no. ADK74340, Phalaenopsis amabilis).

Since a cDNA for KCS, another component of the elongase, was previously obtained as DcKCS1 cDNA from the same carnation cultivar (Harada et al., 2010), the present study completed the identification of each putative cDNA for the four components of FAE in carnation. However, it remains to be elucidated in a future study whether fatty acid elongase complex formation actually occurs only with these four enzyme components or includes another member of the respective enzymes.

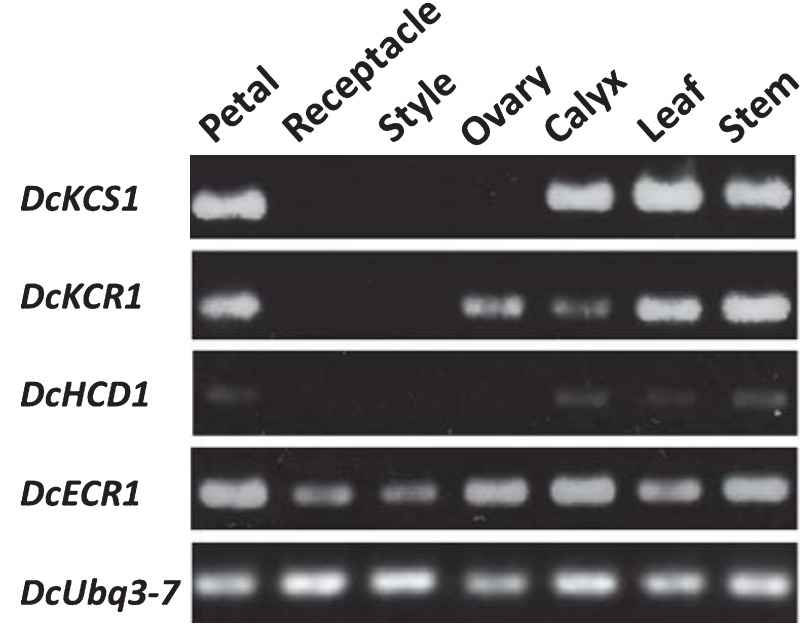

Fig. 3. RT-PCR analysis of the transcripts of DcKCS1, DcKCR1, $D c H C D 1$, and DcECR1 in various tissues of 'Light Pink Barbara' carnation at full-opening stage (Os 6). Ubiquitin (DcUbq 3-7) transcripts were used to ensure equal loading of DNA samples (Nomura et al., 2012).

Expression of DcKCS1, DcKCR1, DcHCD1, and DcECRI in various tissues of carnation

Figure 3 shows the transcript levels of DcKCS1, $D c K C R 1, D c H C D 1$, and DcECR1 in different tissues of carnation plants with opening flowers (Os 6). DcKCSI transcript accumulated in a large amount in petals, calyx, leaves, and stem. DcKCRI transcript accumulated abundantly in petals, leaves, and stem, whereas in a lower amount in the ovary and calyx. DcHCDI transcripts were detected faintly in petals, calyx, leaves, and stem. DcECRI transcripts were detected in all the 
floral and vegetative tissues tested, but in a large amount in petals, ovary, calyx, and stem.

Changes in transcript levels of DcKCS1, DcKCR1, DcHCD1, and DcECRI in carnation petals during flower opening and senescence

Figure 4 shows changes in the transcript level for DcKCS1, DcKCR1, DcHCD1, and DcECR1 relative to that of $D c U b q 3-7$ transcripts in petals of 'Light Pink Barbara' flowers undergoing flower opening and senescence. DcKCS1 transcript levels were low at Os 1 and increased at Os 2 and Os 3, then declined to Os 4 . They again increased at Os 5 and Os 6. The transcript levels decreased to low levels at Ss 2 and Ss 4. This pattern of changes in DcKCS1 transcript levels was similar to that reported previously in opening petals of 'Light Pink Barbara' flowers (Harada et al., 2010). $D c K C R 1$ transcript levels changed similarly to that of $D c K C S 1$, although its transcript level was high even at Os 1. The DcHCD1 transcript level was highest at Os 1 and gradually decreased until Ss 4 , although a slight fluctuation was observed. The DcECRI transcript level was high at Os 1 , and decreased at Os 2, and increased gradually, peaking at Os 5, then declining thereafter.

The present results revealed that DcKCS1 is expressed at high level at Os 2 through Os 6, but not at senescence stages (Ss 2 and Ss 4). Also, DcKCR1, DcHCD1, and $D c E C R 1$, whose translates catalyzed reactions following that by the DcKCS1 translate, were expressed at considerable levels at Os 2 through Os 6. Moreover, the expression of DcKCRI and DcECRI was very low at senescence stages (Ss 2 and Ss 4). These findings indicate that VLCFA synthesis, which is necessary for cuticle wax formation, occurs in petals undergoing expansion growth, resulting in the opening of carnation flowers. It was revealed that KCS, which is responsible for the first step of VLCFA synthesis, is the rate-limiting enzyme of fatty acid elongation and also determines the length of the final acyl-CoA products (Denic and Weissman, 2007; Millar and Kunst, 1997). If this is the case in carnation, the expression of $D c K C S 1$ probably also plays a primary role in VLCFA synthesis in carnation petals.

Comparison of transcripts level in petals of fatty acid elongase components among 'Light Pink Barbara', 'Excerea', and 'Miracle Rouge' carnation

As described in the introduction, cut flowers of 'Miracle Rouge' showed an extremely long vase-life of about three weeks as compared with those of 'Light Pink Barbara' and 'Excerea' cultivars, whose vase-lives were about a week. Repressed ethylene production has been shown to be the primary cause for the long vase-life of senescing flowers in 'Miracle Rouge' flowers (Onozaki et al., 2006; Tanase et al., 2008). In this study, however, we examined other factors, if any, such as cuticle formation in petal cells, which may be related to the long vase-life of senescing flowers in this cultivar. We were interested to know whether the long vase-life of

\section{DcKCS1}

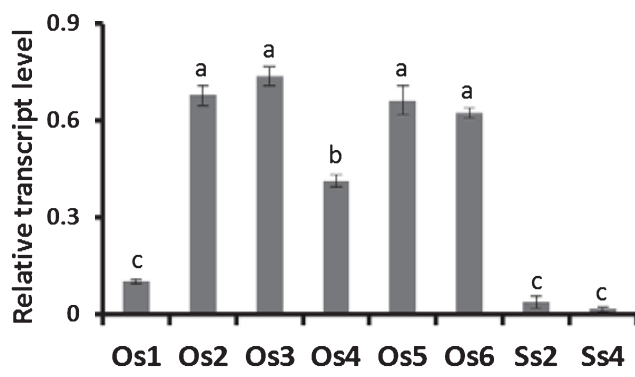

DCHCD1

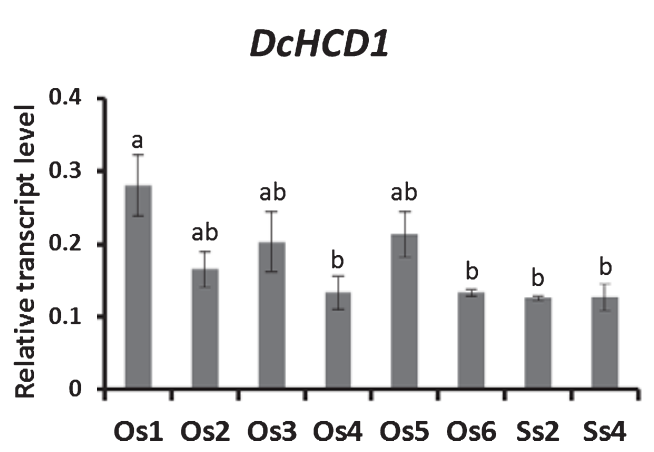

\section{DCKCR1}

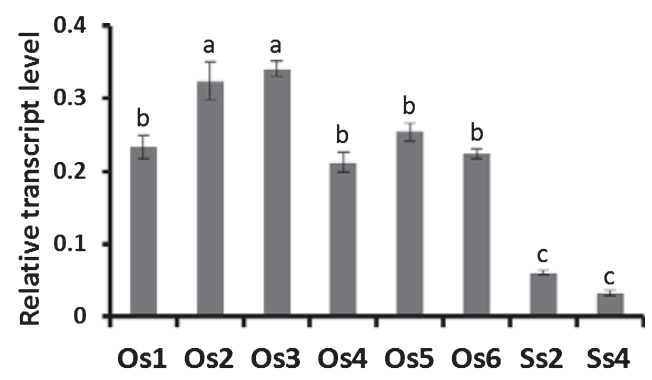

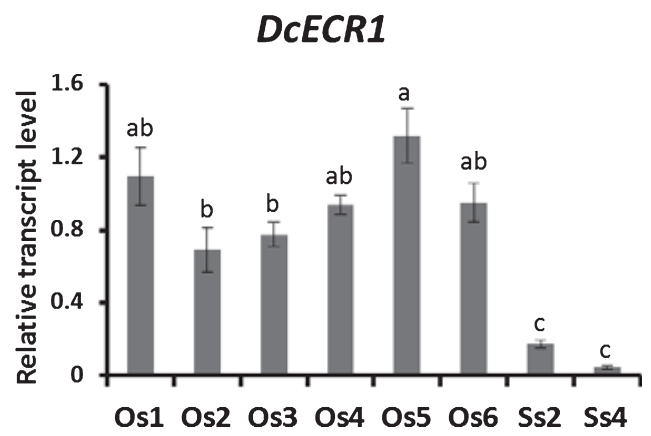

Fig. 4. Real-time RT-PCR analysis of the transcript levels of DcKCS1, DcKCR1, DcHCD1, and DcECR1 in petals of 'Light Pink Barbara' flowers during flower opening and senescence. Relative expression levels were calculated by dividing the transcript copy number of the respective genes with that of $D c U b q 3-7$ transcripts. Data are the means $\pm \mathrm{SE}$ of three separate samples. Vertical bars with different letters are significantly different by Tukey's multiple range test $(p<0.05)$. 
'Miracle Rouge' flowers is related to the active formation of VLCFA synthesis, eventually resulting in a thicker cuticle in this cultivar. A thicker cuticle may prevent water loss from petal surfaces and delay the onset of petal wilting. Flowers of 'Light Pink Barbara', 'Excerea', and 'Miracle Rouge' exhibited a similar time course of flower opening; it took about a week for flower opening from Os 1 to Os 6; therefore, we compared among the three cultivars the cuticle thickness at Os 6 and transcript levels of the four genes in petals. In this experiment, total RNAs were obtained from petal samples of each cultivar, which were prepared by combining equal amount of petals of Os1 to Os 6 (Fig. 5). With all the tree cultivars, cuticles were observed at the arial surface of the adaxial side of petals (Fig. 5A). The cuticle thickness of epidermal cells was $2.37 \mu \mathrm{m}$ for 'Light Pink Barbara', $0.76 \mu \mathrm{m}$ for 'Excerea' and $0.63 \mu \mathrm{m}$ for 'Miracle Rouge' petals (Fig. 5B). These findings revealed that 'Miracle Rouge' petals have a thin cuticle in contrast to our initial speculation. Transcripts of DcKCS1 were detected abundantly in 'Light Pink Barbara', whereas very faintly in 'Excerea' and 'Miracle Rouge' petals (Fig. 5C). With three other genes, $D c K C R 1, D c H C D 1$, and DcECR1, transcripts of all the genes accumulated in 'Light Pink Barbara' petals, whereas only $D c K C R 1$ transcript was detected in 'Excerea' and only DcECRI transcript in 'Miracle Rouge' flowers. The decreased expression of DcKCSI in opening petals of 'Excerea' and 'Miracle Rouge' cultivars may be responsible for the thin cuticle of these two cultivars.

The present findings indicated that the prolonged vaselife of cut flowers of 'Miracle Rouge' is unconnected with the activity of VLCFA synthesis, causing a thick cuticle and thereby preventing water loss during their vase life. However, there may be differences in the shape, surface area or cell-wall thickness of epidermal cells that affect the vase life of cut flowers, among 'Miracle Rouge', 'Light Pink Barbara', and 'Excerea'. These possible differences, if any, were not tested further and remain to be examined in the future. Rather, a different mechanism for the prolonged vase life of 'Miracle Rouge' flowers should be researched. Nomura et al. (unpublished) are investigating the involvement of plant hormones, other than ethylene, in depressed ethylene production, leading to the prolonged vase life of 'Miracle Rouge' flowers.

In conclusion, the present study isolated cDNAs for
A.

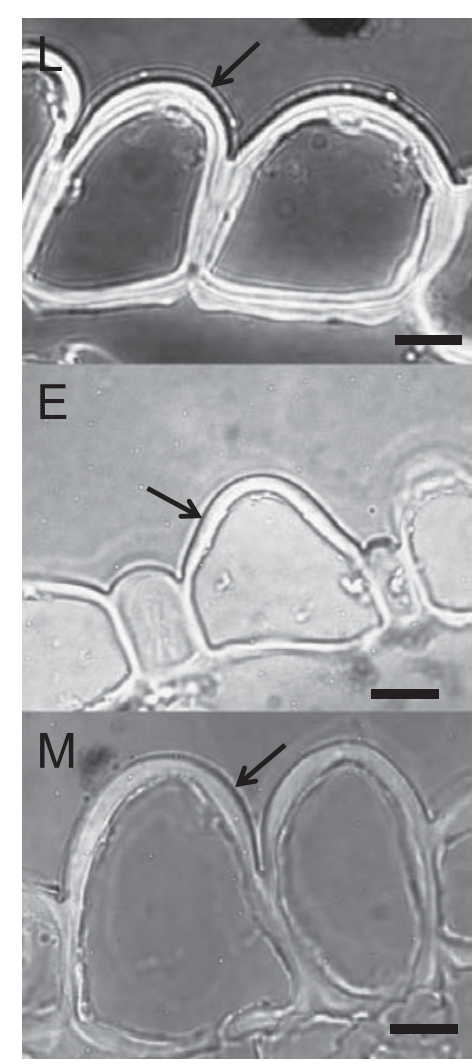

B.

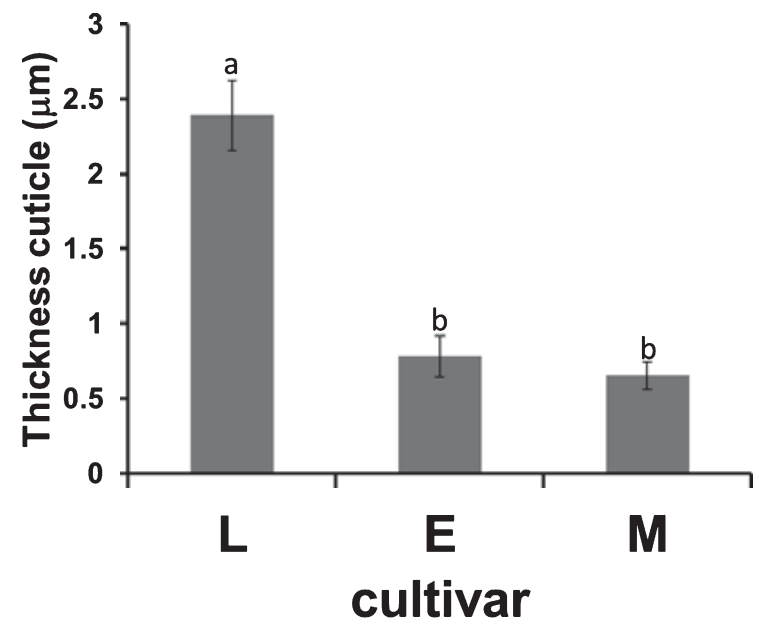

C.

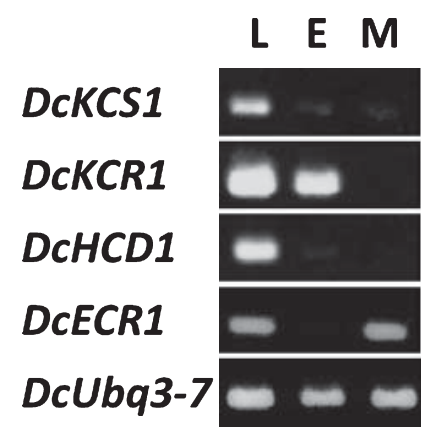

Fig. 5. Comparison of cuticle appearance (A), its thickness (B), and transcript levels (C) of DcKCS1, DcKCR1, DcHCD1, and $D c E C R 1$ among 'Light Pink Barbara (L)', 'Excerea (E)', and 'Miracle Rouge (M)'. Cuticle thickness was determined with petals at Os 6, and data are the mean \pm SE of measurements of ten epidermal cells on the adaxial side. Transcript levels were determined with total RNA obtained from petal samples, which were made by mixing equal amounts of petals detached from flowers from Os 1 to Os 6 . Vertical bars with different letters were significantly different by Tukey's multiple range test $(p<0.05)$. Bars are $20 \mu \mathrm{m}$. Arrows show cuticle layer after staining. 
DcKCR1, DcHCD1, and DcECRI and, combined with the previously isolated DcKCSI cDNA (Harada et al., 2010), completed the possible identification of the respective cDNAs of four enzymes composed of FAE in carnation petals. Furthermore, this study showed that the expressions of the four genes are active during the flower-opening stage, which is concomitant with the expansion growth of petals, requiring the rapid formation of waxy cuticle; however, the prolonged vase life of 'Miracle Rouge' flowers seemed not to be related to repressed cuticle formation.

\section{Literature Cited}

Abeles, F. B., P. W. Morgan and M. E. Saltveit, Jr. 1992. Ethylene in Plant Biology. 2nd ed. Academic Press, San Diego.

Bach, L., L. V. Michaelson, R. Haslam, Y. Bellec, L. Gissot, J. Marion, M. Da Costa, J. Boutin, M. Miquel, F. Tellier, F. Domergue, J. E. Markham, F. Beaudoin, J. A. Napier and J-D. Faure. 2008. The very-long-chain hydroxy fatty acylCoA dehydratase PASTICCINO2 is essential and limiting for plant development. Proc. Natl. Acad. Sci. USA 105: 14727 14731.

Beaudoin, F., X. Wu, F. Li, R. P. Haslam, J. E. Markham, H. Zheng, J. A. Napier and L. Kunst. 2009. Functional characterization of the Arabidopsis thaliana $\beta$-ketoacyl-CoA reductase candidates of the fatty acid elongase. Plant Physiol. 150: 1174-1191.

Borochov, A. and W. R. Woodson. 1989. Physiology and biochemistry of flower petal senescence. Hort. Rev. 11: 1543.

Denic, V. and J. S. Weissman. 2007. A molecular caliper mechanism for determining very long-chain fatty acid length. Cell 130: 663-677.

Frohman, M. A., M. K. Dush and G. R. Martin. 1988. Rapid production of full-length cDNAs from rare transcripts: amplification using a single gene-specific oligonucleotide primer. Proc. Natl. Acad. Sci. USA 85: 8998-9002.

Goodwin, S. M., N. Kolosova, C. M. Kish, K. V. Wood, N. Dudareva and M. A. Jenks. 2003. Cuticle characteristics and volatile emission of petals in Antirrhinum majus. Physiol. Plant. 117: 435-443.

Harada, T., S. Satoh, T. Yoshioka and K. Ishizawa. 2005. Expression of sucrose synthase genes involved in enhanced elongation of pondweed (Potamogeton distinctus) turions under anoxia. Ann. Bot. 96: 683-692.

Harada, T., Y. Torii, S. Morita, T. Masumura and S. Satoh. 2010. Differential expression of genes identified by suppression subtractive hybridization in petals of opening carnation flowers. J. Exp. Bot. 61: 2345-2354.

Harwood, J. L. 1997. Plant lipid metabolism. p. 259-271. In: P. M. Dey and J. B. Harborne (eds.). Plant Biochemistry. Academic Press Ltd., San Diego.
Kawamoto, T. 2003. Use of a new adhesive film for the preparation of multi-purpose fresh-frozen sections from hard tissues, whole animals and plants. Arch. Hist. Cytol. 66: 123-143.

Kunst, L. and L. Samuels. 2009. Plant cuticles shine: advances in wax biosynthesis and export. Cur. Opin. Plant Biol. 12: 721727.

Millar, A. A. and L. Kunst. 1997. Very-long-chain fatty acid biosynthesis is controlled through the expression and specifity of the condensing enzyme. Plant J. 12: 121-123.

Millar, A. A., S. Clemens, S. Zachgo, E. M. Giblin, D. C. Taylor and L. Kunst. 1999. CUT1, an Arabidopsis gene required for cuticular wax biosynthesis and pollen fertility, encodes a verylong-chain fatty acid condensing enzyme. Plant Cell 11: 825838.

Morita, S., Y. Torii, T. Harada, M. Kawarada, R. Onodera. and S. Satoh. 2011. Cloning and characterization of a cDNA encoding sucrose synthase associated with flower opening through early senescence in carnation (Dianthus caryophyllus L.). J. Japan. Soc. Hort. Sci. 80: 358-364.

Nomura, Y., S. Morita, T. Harada and S. Satoh. 2012. Cloning, characterization and expression of carnation (Dianthus caryophyllus L.) ubiquitin genes and their use as a normalization standard for gene expression analysis in senescing petals. J. Japan. Soc. Hort. Sci. 81: 357-365.

Onozaki, T., H. Ikeda, M. Shibata, N. Tanikawa, M. Yagi, T. Yamaguchi and M. Amano. 2006. Breeding and characteristics of carnation Norin No.1 'Miracle Rouge' and No.2 'Miracle Symphony' with long vase life. Bul. Natl. Inst. Floric. Sci. 5: 1-16 (In Japanese with English abstract).

Raffaele, S., F. Vailleau, A. Leger, J. Joubes, O. Miersch, C. Huard, E. Blee, S. Mongrand, F. Domergue and D. Roby. 2008. A MYB transcription factor regulates very-long-chain fatty acid biosynthesis for activation of the hypersensitive cell death response in Arabidopsis. Plant Cell 20: 752-767.

Reid, M. S. and M-J. Wu. 1992. Ethylene and flower senescence. Plant Growth Regul. 11: 37-43.

Riederer, M. and L. Schreiber. 2001. Protecting against water loss: analysis of the barrier properties of plant cuticles. J. Exp. Bot. 52: 2023-2032.

Shibuya, K., T. Yoshioka, T. Hashiba and S. Satoh. 2000. Role of the gynoecium in natural senescence of carnation (Dianthus caryophyllus L.) flowers. J. Exp. Bot. 51: 2067-2073.

Tanase, K., T. Onozaki, S. Satoh, M. Shibata and K. Ichimura. 2008. Differential expression levels of ethylene biosynthetic pathway genes during senescence of long-lived carnation cultivars. Postharvest Biol. Technol. 47: 210-217.

ten Have, A. and E. J. Woltering. 1997. Ethylene biosynthetic genes are differentially expressed during carnation (Dianthus caryophyllus L.) flower senescence. Plant Mol. Biol. 34: 8997.

Zheng, H., O. Rowland and L. Kunst. 2005. Disruptions of the Arabidopsis enoyl-CoA reductase gene reveal an essential role for very-long-chain fatty acid synthesis in cell expansion during plant morphogenesis. Plant Cell 17: 1467-1481. 